\title{
A modified Delphi to define drug dosing errors in pediatric critical care
}

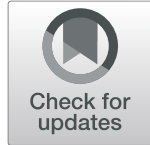

Nadia Roumeliotis ${ }^{1,2,3^{*}}$ (D) Eleanor Pullenayegum ${ }^{2,3}$, Paula Rochon ${ }^{4,5,6}$, Anna Taddio ${ }^{2,7}$ and Chris Parshuram ${ }^{1,2,3,8}$

\begin{abstract}
Background: There is no globally accepted definition for dosing error in adult or pediatric practice. The definition of pediatric dosing error varies greatly in the literature. The objective of this study was to develop a framework, informed by a set of principles, for a clinician-based definition of drug dosing errors in critically ill children, and to identify the range that practitioners agree is a dosing error for different drug classes and clinical scenarios.

Methods: We conducted a nationwide three staged modified Delphi from May to December 2019. Expert clinicians included Canadian pediatric intensive care unit (PICU) physicians, pharmacists and nurses, with a least 5 years' experience. Outcomes were underlying principles of drug dosing, and error thresholds, as defined by proportion above and below reference range, for common PICU medications and clinical scenarios.

Results: Forty-four participants met eligibility, and response rates were 95, 86 and $84 \%$ for all three rounds respectively. Consensus was achieved for 13 of 15 principles, and 23 of 30 error thresholds. An over-dosed drug that is intercepted, an under-dose of a possibly life-saving medication, dosing $50 \%$ above or below target range and not adjusting for a drug interaction were agreed principles of dosing error. Altough there remained much uncertainty in defining dosing error, expert clinicians agreed that, for most medication categories and clinical scenarios, dosing over or below $10 \%$ of reference range was considered an error threshold.

Conclusion: Dosing principles and threshold are complex in pediatric critical care, and expert clinicians were uncertain about whether many scenarios were considered in error. For most intermittent medications, dosing over $10 \%$ below or above reference range was considered a dosing error, although this was largely influenced by clinical context and drug properties. This consensus driven error threshold will help guide routine clinical dosing practice, standardized reporting and drug quality improvement in pediatric critical care.
\end{abstract}

Keywords: Dosing, Error, Pediatrics, Critical care

\section{Background}

Dosing errors are the amongst the most common types of medication error in pediatric practice, along with incomplete and incorrect prescription preparation [1-6]. .Pediatric drug dosing is particularly challenging due to a child's growth, development and change in organ

\footnotetext{
* Correspondence: nadia.roumeliotis@gmail.com

'Department of Critical Care Medicine, The Hospital for Sick Children, 555 University Avenue, Toronto, ON, Canada

${ }^{2}$ Child Health Evaluative Sciences, SickKids Research Institute, Toronto, Canada

Full list of author information is available at the end of the article
}

function over time. Dosing in children is therefore weight-based, involves the dilution of concentrated stock solutions, and the off-label use of medications approved for adults [7]. Despite standard weight-based dosing in pediatrics, doses used in clinical practice can vary between practitioners, clinical settings, and healthcare centers. Dosing is often based on patient characteristics and clinician comfort [8], due to a lack of established evidence based guidelines for appropriate pediatric dosing of off-label drugs in children. Critically ill children present a further challenge for dosing due to the pharmacokinetic changes associated with concurrent organ

(c) The Author(s). 2020 Open Access This article is licensed under a Creative Commons Attribution 4.0 International License which permits use, sharing, adaptation, distribution and reproduction in any medium or format, as long as you give appropriate credit to the original author(s) and the source, provide a link to the Creative Commons licence, and indicate if changes were made. The images or other third party material in this article are included in the article's Creative Commons licence, unless indicated otherwise in a credit line to the material. If material is not included in the article's Creative Commons licence and your intended use is not permitted by statutory regulation or exceeds the permitted use, you will need to obtain permission directly from the copyright holder. To view a copy of this licence, visit http://creativecommons.org/licenses/by/4.0/ The Creative Commons Public Domain Dedication waiver (http://creativecommons.org/publicdomain/zero/1.0/) applies to the data made available in this article, unless otherwise stated in a credit line to the data. 
dysfunction and acuity of illness, as well as the pharmacodynamic properties associated with the concomitant exposure of other potentially harmful medications.

There is no consensus definition for dosing error in adult or pediatric practice [9]. Although a clinicianbased definition for prescribing error has been proposed in pediatrics $[8,10]$, no such definition exists specifically exists for dosing error. Heterogenous definitions of pediatric dosing error in the literature include an "error in drug dosing, ordering, transcribing, administering or monitoring" [1, 4, 11, 12]; a dosing error "higher than reference dosing" [3]; "over 10\% difference from reference dosing" [13]; "over $20 \%$ dose deviation from recommended dose" [7], +/-25\% of recommended dose (Ghaleb) or pharmacist consensus [14].

In clinical practice, critical care practitioners may prescribe acceptable doses outside a reference range, based on drug properties, organ dysfunction, and the patients' clinical status. Dosing references and a more concrete dosing error definition are still needed however, to guide standardized error reporting, quality improvement for optimal prescribing practices, error comparisons within research and clinical decision support tools. We sought to better understand what constitutes a dosing error in the pediatric intensive care unit (PICU). The specific objectives of this study were 1) to develop a framework, informed by a set of principles, for a clinician-based definition of drug dosing errors in critically ill children, and 2) to identify the range, outside the standard reference range, that practitioners agree is a dosing error for different drug classes and clinical scenarios.

\section{Methods}

\section{Design}

We conducted a nationwide, three staged, modified Delphi method from May to December 2019, in Canada. We developed a detailed survey eliciting the opinions of PICU healthcare providers on 1) the underlying principles that guide dosing and influence the consideration of dosing error, and 2) dosing thresholds for dosing errors, in pediatric critical care. The study reporting followed the STROBE guidelines (STROBE checklist, Supplement Table 1) [15].

The Delphi methodology was used to achieve consensus amongst a group of PICU experts, with multiple iterative rounds and anonymous feedback after each round [16]. This methodology was favoured over other structured consensus methods as it did not require faceto-face group interaction, was questionnaire based, preserved anonymity of respondents, and has been frequently used and accepted for scientific consensus of this nature $[17,18]$. Furthermore, the electronic distribution of the survey allowed for the timely completion of a nationwide survey with multiple rounds for participants.
Given that this Delphi was conducted across Canada, we opted for a modified Delphi approach. A modified Delphi differs from the classical Delphi method in that the expert panel was not involved in the initial process of generating principles; this initial step was replaced by local face-to-face focus groups [19].

\section{Target population}

We conducted deliberate purposive sampling of expert clinicians across Canada. Eligible clinicians were physicians, nurse managers and pharmacists who were currently working the majority of their time in a Canadian PICU, with at least 5 years PICU experience. Participants were contacted through the electronic mailing list of the Canadian Critical Care Trials Group (CCCTG), asking for their interest in participating. From this mailing list, we then further attempted to solicit at least one eligible physician, nurse and pharmacist from each center, by asking CCCTG members to refer participants who may be interested. Participation on the expert panel was voluntary. No incentives were offered.

An a priori sample size of 40 expert healthcare providers was established $[8,10,17,18]$. This is slightly higher than previous pharmacological studies using Delphi consensus methodology. Assuming an $80 \%$ response rate and loss of adherence through three rounds, recruitment was maintained open until at least 50 providers agreed to participate.

\section{Outcomes}

Two outcomes were assessed: dosing principles and error thresholds. Dosing principles were elicited by literature review and small local focus groups, and then measured by the expert panel with the strength of agreement and disagreement on a Likert scale. Error thresholds were defined as the proportion above and below reference range that expert clinicians agreed was a dosing error, given the specific drug class and clinical context.

\section{Intervention}

\section{Survey creation - item generation \& reduction}

The survey was generated using a guide for selfadministered surveys of clinicians [20]. Survey principles were generated through literature review, and expert opinion in small local focus group sessions. Two small multidisciplinary focus groups were held with target clinicians $(n=9$, including physicians, pharmacists and nurses) in order to brainstorm factors that may affect dosing threshold. Groups were continued until no new items were generated, and dosing threshold cut-offs were established. The rationale for the medication classes tested in the survey were based on the medications with the most frequent dosing errors in pediatrics; anti- 
infectives, analgesic and sedative agents, and electrolytes $[1,6,7]$. Item reduction was achieved by eliminating superfluous questions to minimize respondent burden, while retaining items that addressed a variety of medications classes, adjustment for organ dysfunction and pharmacokinetic properties. Further items were removed and/or altered after pre-testing the survey amongst clinicians. Error thresholds were established with focus group consensus and translated to a Likert scale (See Supplement Figure 1). Three of the nine clinicians from the focus groups went on to participate in the Delphi.

\section{Survey format}

The survey was divided into three sections; demographic description of respondents, dosing principles and error thresholds. Dosing principles included drug, patient and clinical factors and were scored on a 5-point Likert scale from "Yes, definitely an error" to "No, definitely not an error" (See Supplement Table 2). The dosing error thresholds were on a 4 - or 5 - point Likert scale. There were 4 thresholds categories for under-dosing (1-10\%, $11-20 \%, 21-50 \%, 51-100 \%)$ and 5 for overdosing (1$10 \%, 11-20 \%, 21-50 \%, 51-100,>100 \%)$. The option for "I don't know" was always available to acknowledge uncertainty, as was the option "Depends" to identify new issues or principles that may suggest an error in one type of clinical scenario, but not another. An example of an error threshold round 2 question is included in Figure 1 in the Supplement.

\section{Testing}

Pre-testing of the survey was conducted with the help of clinicians in 2 different PICUs to aid in refining questionnaire content and the format of answers. Pilot testing, conducted in 2 centers, evaluated clinical sensibility including: face validity; content validity, whether questionnaire was measuring what it was intended to measure, clarity and comprehension.

\section{Survey administration}

Expert clinician participants were recruited via e-mail. Upon agreement to participate, an electronic survey or paper survey was sent, according to respondent preference and the desire to preserve anonymity. All surveys were anonymous and consent was implicit upon survey completion. Clinicians who responded but were ineligible, were not included in analysis or further rounds. Participants received 3 reminders to complete each round. In rounds 2 and 3 of the survey, questions where consensus was achieved were removed, and aggregated responses of participants were included for questions where consensus was not achieved.

\section{Data analysis}

Data were summarized by numbers and proportions, and reported according to proposed methodological criteria for Delphi [21]. An a priori definition of consensus was established. Consensus was achieved when $>=70 \%$ of respondents agreed on an answer in Round 1, and $>=$ $60 \%$ of respondents agreed to an answer in Round 2 and Round 3 [21]. Disagreement was defined as $35 \%$ or more of responses falling in both of the 2 extreme ranges of options on the Likert scale. All other combinations of panel answers were considered as 'partial agreement'. Blank responses were entered as blank, and proportions were adjusted accordingly. Data from surveys were imported and analyzed with Excel version 16.2. Research Ethics Board Approval was obtained from the Hospital for Sick Children (\# 1000062863) and the University of Toronto (\#00037969).

\section{Results}

We invited 82 clinicians to participate in the study, of which 51 (62\%) agreed to participate. Of these, 44 (89\%) providers met eligibility criteria, and were invited to complete further rounds. Figure 1 illustrates the flow chart of the modified Delphi process. Response rates for Rounds 1, 2 and 3 were 95, 86 and 84\% respectively. Expert clinicians came from 14 pediatric centers in 6 Canadian provinces.

Of the 44 included expert clinicians, 42 completed round 1; 26 (62\%) physicians, 13 (31\%) pharmacists and $3(7 \%)$ nurses. The providers prescribed medication occasionally (1-5 times/month) or daily in 11 and 26 cases, and administered medication never or occasionally $(<6$ times per month) in 14 and 25 cases respectively. The demographic information on participants, for all three rounds, is included in Table 1.

The most frequently reported resources used for pediatric dosing were: the local hospital formulary $(N=$ $38)$, Lexi-comp on-line (N-34), memorized doses $(N=$ $30)$, local hospital pharmacist $(N=29)$, and local ICU guidelines $(N=22)$. Less frequently used resources included the electronic Compendium of Pharmaceuticals and Specialties (e-CPS) $(N=4)$, UpToDate $(N=3)$, and PubMed $(N=1)$.

\section{Principles of dosing errors}

Table 2 presents the 15 different patient-, drug- and dosing- principles evaluated, the agreement statement and the proportion of consensus agreement for each principle. Consensus was achieved for 13 of 15 principles: 2 principles were rated as "definite dosing error", 3 were rated as "probable dosing error", 7 as "may or may not be a dosing error", 1 as "probably not a dosing error", and 2 with partial agreement achieved. No principles were considered "definitely not dosing 


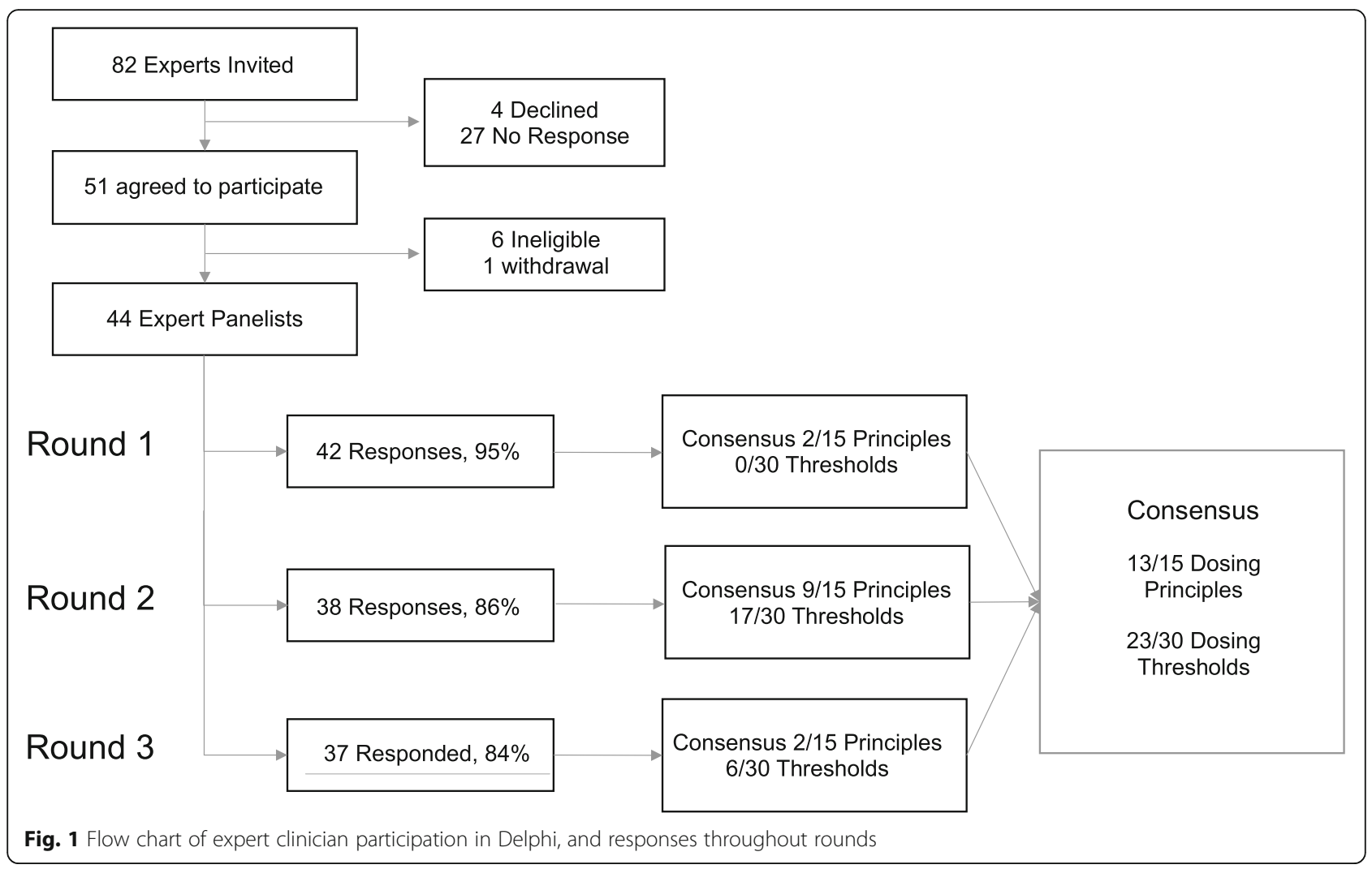

error". An 'over-dosed drug that is intercepted', and an 'administered under-dose of a possibly life-saving medication', were agreed to be definite dosing errors. Experts agreed that 'dosing 50\% above or below target recommended range and not adjusting for a drug interaction' was a probable dosing error. Experts also agreed on principles which may or may not constitute a dosing error; including 'weight-based dosing without accounting for age, body surface area, renal insufficiency', and 'dosing above maximum recommended dosing' (whether on Extracorporeal Membrane Oxygenation (ECMO), end of life, or neither). The only statement that experts felt was unlikely to be an error was 'dosing a drug that cannot easily be administered in dosage forms available'. There were no statements that experts felt definitely did not constitute a dosing error. The principle addressing drug dosing after a previous adverse drug event (ADE), and the principle regarding off-label drug dosing, did not achieve consensus.

\section{Error thresholds}

We evaluated 15 above and below dosing error thresholds ( $N=30$ error thresholds). Consensus was achieved for 23 thresholds; 12 under-dose error thresholds and 11 overdose error thresholds. Progression of the consensus over 3 rounds is presented in Fig. 1. Where consensus was achieved, expert providers agreed, for most medication categories and clinical scenarios that dosing over or below $10 \%$ of reference range was considered an error (category $11-20 \%$ was an error). Results of the error thresholds are presented in Table 3.

For nephrotoxic antibiotics (such as gentamicin and vancomycin), $10 \%$ above and below reference range, when renal function was adjusted for, was considered an error. For hepatotoxic medications (such as acetaminophen and NSAIDs), 10\% above and below reference range, when hepatic function was adjusted for, was also considered an error. Error thresholds for opioid and benzodiazepines over-dose error were $10 \%$ above reference range regardless of presence of renal failure, as long as dose was renally adjusted. When the scenario included opioid tolerance there was consensus on the lower threshold but only partial agreement on the upper threshold for dosing (dosing $>10 \%$ remained the most common choice $n=19(50 \%)$ ).

For non-toxic antibiotics (such as Ampicillin), partial agreement of over $50 \%$ expert providers considered > $20 \%$ above reference range as an error and $10 \%$ below reference to be an error (consensus).

In all three scenarios where a previous adverse drug event had occurred with the same drug, experts never achieved consensus on the upper threshold for error, and were more conservative in their dosing. In a number 
Table 1 Demographics of included expert participants

\begin{tabular}{|c|c|c|c|}
\hline & $\begin{array}{l}\text { Round } 1 \\
N=42, \mathrm{n}(\%)\end{array}$ & $\begin{array}{l}\text { Round } 2 \\
N=38, \mathrm{n}(\%)\end{array}$ & $\begin{array}{l}\text { Round } 3 \\
N=37, \mathrm{n}(\%)\end{array}$ \\
\hline \multicolumn{4}{|c|}{ Healthcare Providers } \\
\hline Physician & $26(62)$ & $23(61)$ & $24(65)$ \\
\hline Pharmacist & $13(31)$ & $13(34)$ & $11(30)$ \\
\hline Nurse & $3(7)$ & $2(5)$ & $2(5)$ \\
\hline \multicolumn{4}{|c|}{ Length of Practice in PICU } \\
\hline $5-10$ years $^{\mathrm{a}}$ & $18(43)$ & $15(39)$ & $15(41)$ \\
\hline $10-15$ years & $7(17)$ & $12(32)$ & $10(27)$ \\
\hline$>15$ years & $17(40)$ & $11(29)$ & $12(32)$ \\
\hline \multicolumn{4}{|l|}{ Size of PICU } \\
\hline$<10$ beds & $3(7)$ & $3(8)$ & $3(8)$ \\
\hline $10-15$ beds & $16(38)$ & $11(19)$ & $13(35)$ \\
\hline $16-20$ beds & $7(17)$ & $8(21)$ & $5(14)$ \\
\hline $21-25$ beds & $6(14)$ & $4(10)$ & $3(8)$ \\
\hline$>25$ beds & $10(24)$ & $12(32)$ & $13(35)$ \\
\hline \multicolumn{4}{|l|}{ Province } \\
\hline British-Columbia & $3(7)$ & $3(8)$ & $2(5)$ \\
\hline Alberta & $3(7)$ & $2(5)$ & $2(5)$ \\
\hline Manitoba & $4(10)$ & $2(5)$ & $3(8)$ \\
\hline Ontario & $14(33)$ & $13(34)$ & $16(43)$ \\
\hline Quebec & $17(40)$ & $17(40)$ & $13(35)$ \\
\hline Nova-Scotia & $1(2)$ & $1(3)$ & $1(3)$ \\
\hline
\end{tabular}

PICU Pediatric Intensive Care Unit

${ }^{a} 1$ participant with 5 years' experience (including fellowship) was included

of cases $(N=6)$, clinicians said they would not give the drug to a patient with a previous serious adverse drug event. Many others answered 'Depends' and suggested that the circumstances of the ADE where important to consider: nature and severity of the ADE, whether it was allergic or not, the concomitant medications, and the balance of desirable versus undesirable side effects. This finding is consistent with the answers on the dosing principles as consensus was also not achieved when evaluating a dose where a previous ADE has occurred. In medications with a high potential toxicity (e.g. potassium chloride), clinicians were also unwilling to exceed reference dosing (partial agreement, $N=21 / 37$ (56\%)).

Sixty-six percent of clinicians said over-dosing was not equivalent to under-dosing; and this was justified in comments mostly relating to the effects of over-dose vs under-dose.

\section{Discussion}

This study used a modified Delphi methodology to establish a framework of principles and dosing error thresholds for drugs commonly given in the PICU under common clinical scenarios. A group of 44 PICU clinicians were recruited from across Canada with participation in all three rounds exceeding $80 \%$. Clinicians agreed that under-dosing potentially life-saving drugs in the PICU is a definite error, as were near-miss over-doses. Under-dosing and over-dosing expected to give low or high serum concentration was also a probable error. There were many scenarios however, where clinicians agreed that establishing error was more difficult and complex. These scenarios include weight-based dosing without accounting for age, body surface area, or renal dysfunction, and dosing above the maximum limit in ECMO or end-of-life.

Expert PICU clinicians agreed that, for most drugs in most clinical scenarios, dosing more than $10 \%$ above and below reference range was an error threshold. This was internally consistent with the principle of over and under-dosing described above, as well as commonly accepted practice amongst many pharmacists. There were however, scenarios where defining error was difficult despite consensus; including adjusting dosing for age, body mass index, renal impairment, and exceeding maximum dosing on ECMO, in end of life care or if the drug is 'life saving' (Table 2). The consensus achieved in the ambiguity of defining these scenarios as errors, reflects the complexity that prescriber experience, intent and patient clinical context may render excess dosing as acceptable. There were other scenarios where consensus could not be reached; including all clinical scenarios where a previous ADE had occurred. Although some providers adjusted their error thresholds to more narrow dosing, others simply would not administer the drug after a harmful ADE.

Responses in dosing error thresholds were mostly consistent with the consensus on the dosing principles. There were however, scenarios where clinicians were not completely consistent in their choices: they agreed that 'dosing 50\% above or below target range and not adjusting for a drug interaction' was a probable dosing error, despite the fact that most chose a $10 \%$ overdose threshold. The choice of 'probable', 'definite' and 'may or may not be' a dosing error, suggests that clinicians were able to potentially conceive of instances where selective overdosing might not be an error, and reflects the complexity of defining error.

Despite clinicians selecting the same thresholds for many medication groups and clinical scenarios, they were able to modify and adapt their responses to different clinical scenarios. This adaptability is evidenced by the lower threshold selected for the anticoagulant dosing with previous adverse event (1\% threshold versus $10 \%$ when ADE had occurred).

The results of this study reflect the complexity of dosing in clinical practice and the difficulty in establishing a definition. Participants were uncertain as to whether many clinical scenarios constituted dosing errors 
Table 2 Principles of dosing error, agreement statement, and proportion of consensus agreement ${ }^{\mathrm{a}}$ for each statement

\begin{tabular}{ll}
\hline & $\begin{array}{l}\text { Proportion of } \\
\text { Round agre }\end{array}$ \\
\hline YES, DEFINITELY constitutes a dosing error & $\mathbf{7 6}$ Round 1 \\
Dosing a drug above reference range, but the drug dose was intercepted (near-miss) and did not reach patient & $\mathbf{7 3}$ Round 2 \\
Dosing a drug below the minimum recommended dose, when the drug is possibly life-saving & $\mathbf{6 2}$ Round 2 \\
YES, PROBABLY constitutes a dosing error & $\mathbf{6 8}$ Round 3 \\
Dosing a drug in a dose that is predicted to give serum levels below desired therapeutic range (e.g. 50\% below \\
lower limit target) & $\mathbf{6 0}$ Round 2 \\
Dosing a drug by weight, without accounting for a significant drug interaction & $\mathbf{7 6}$ Round 2 \\
Dosing a drug in a dose that is likely to give serum levels above desired therapeutic range (e.g. 50\% above upper \\
limit target) & $\mathbf{7 3}$ Round 1 \\
MAY or MAY NOT be a dosing Error & $\mathbf{6 6}$ Round 2 \\
Dosing a drug by weight, without adjusting for age & $\mathbf{7 1}$ Round 2 \\
Dosing a drug by weight, without accounting for body surface area & $\mathbf{8 2}$ Round 2 \\
Dosing a drug dose above the maximum dose recommended in the hospital formulary, guideline or reference \\
sources & $\mathbf{6 0}$ Round 2 \\
Dosing a drug above the maximum recommended dose, in a patient who is in end-of life care & $\mathbf{6 9}$ Round 3 \\
Dosing a drug above the maximum recommended dose in a patient who is on ECMO & \\
Dosing a drug above the maximum recommended dose, when the drug is possibly life-saving (e.g. antibiotics & \\
in sepsis) & \\
Dosing a drug by weight, without adjusting for renal insufficiency (Creatinine $50 \%$ higher than baseline) &
\end{tabular}

NO, UNLIKELY to constitute a dosing error

Dosing a drug dose that cannot easily be administered using dosage forms available

66 Round 2

\section{NO, DEFINITELY NOT a dosing error}

\section{None}

Dosing a drug at a dose where the patient has had a previous severe adverse event

Dosing a drug for which the use is off-label in children (i.e. prescribed for a condition that it is not officially approved for)

$\mathrm{N}$ for Round 1 is $42, \mathrm{~N}$ for Round $37, \mathrm{~N}$ for Round 3 is 36

${ }^{a}$ Consensus agreement established a priori was $>=70 \%$ for Round 1, and $>=60 \%$ for Rounds 2 and 3.

${ }^{b}$ ECMO: Extra-Corporeal Membrane Oxygenation.

" $N=21$ (58\%) responded "Yes, definitely dosing error", $N=1$ "Yes, probable dosing error" and $N=14$ (39\%) "May or May not be error". N total = 36 participants.

${ }^{\mathrm{d}} N=20$ (55\%) "No Definitely not a dosing error", $N=12$ (33\%) "No, probably not a dosing error", $N=3$ ( $8 \%$ ) "May or may not be a dosing error", $N=1$ (3\%) "I don't know". $\mathrm{N}$ total $=36$ participants.

(Table 2). That being said, there is a need for a more pragmatic definition of dosing error. Drug dosing error evaluation (causes, etiology, related adverse events) first requires defining whether an error has occurred to establish preventability; adverse events may occur with an appropriately dosed drug, or with an inappropriately dosed drug (under- or over-dosed). Although Ghaleb et al. evaluated principles of prescription error in pediatrics [8], there definition contains subjective terms related to the significance and harm related to an error. Within regards to dosing, consensus was achieved amongst their participants that dosing a drug above or below $25 \%$ of the reference range should be an error [8]. A recent pediatric study by Howlett et al. used a Delphi method to identify medication errors related the health information systems, but these did not address dosing ranges [18]. The current heterogeneity in defining dosing error in the literature makes comparison of results across studies of medication error very difficult, and fails to adequately define optimal prescribing practices. This study will help define dosing error thresholds for the prescription of intermittent drugs in routine clinical practice and future research studies, as defined by expert specialists -filling a gap in the pediatric dosing literature. These consensus-based results may facilitate measurement and research comparisons, along with providing guidance for quality improvement initiatives and optimal prescribing, standardized error reporting, and building decision support tools with health system informatics.

This study has a few limitations. First, participants in this study may not adequately reflect other PICU providers, both within and outside Canada - therefore generalizability may be limited. That being said, limited 
Table 3 Proportion below and above reference range dosing that expert participants would consider is a dosing error, and proportion of consensus agreement

\begin{tabular}{|c|c|c|}
\hline Drug Class and Patient characteristics & $\begin{array}{l}\text { Threshold for dosing error below } \\
\text { reference range ( } \% \text { consensus) }\end{array}$ & $\begin{array}{l}\text { Threshold for dosing error Above } \\
\text { reference range (\% consensus) }\end{array}$ \\
\hline \multicolumn{3}{|l|}{ NEPHROTOXIC ANTIBIOTIC (e.g. Gentamicin, Vancomycin) } \\
\hline In a patient with normal renal function ${ }^{a}$ & $10 \%(81)$ & $10 \%(79)$ \\
\hline In a patient with acute renal failure ${ }^{b}$ & $10 \%(76)$ & $10 \%(74)$ \\
\hline \multicolumn{3}{|l|}{ NON-TOXIC ANTIBIOTIC (e.g. Ampicillin) ${ }^{a}$} \\
\hline In stable patient, no organ dysfunction & $10 \%(68)$ & Partial agreement ${ }^{c}$ \\
\hline \multicolumn{3}{|l|}{ HEPATOTOXIC MEDICATION (e.g. Acetaminophen) } \\
\hline In patient with normal hepatic function & $10 \%(74)$ & $10 \%(76)$ \\
\hline In a patient with acute hepatic failure & $10 \%(76)$ & $10 \%(74)$ \\
\hline \multicolumn{3}{|l|}{ OPIOID (e.g Morphine) } \\
\hline In an opioid naïve patient with normal organ function & $10 \%(66)$ & $10 \%(71)$ \\
\hline In a patient with an opioid tolerance & $10 \%(63)$ & Partial agreement $^{d}$ \\
\hline In a patient with acute renal failure ${ }^{b}$ & $10 \%(74)$ & $10 \%(79)$ \\
\hline In a patient with a previous opioid related adverse event ${ }^{\mathrm{h}}$ & Partial agreement $\mathrm{e}^{\mathrm{e}}$ & Partial agreement $\mathrm{e}^{\mathrm{e}}$ \\
\hline \multicolumn{3}{|l|}{ BENZODIAZEPINE (e.g. Lorazepam) } \\
\hline In an opioid naïve patient with normal organ function & $10 \%(61)$ & $10 \%(79)$ \\
\hline In a patient with acute renal failure ${ }^{b}$ & $10 \%(66)$ & $10 \%(84)$ \\
\hline In a patient with a previous opioid related adverse event ${ }^{h}$ & Partial agreement ${ }^{\mathrm{f}}$ & $10 \%(60)$ \\
\hline \multicolumn{3}{|l|}{ ANTICOAGULANT (e.g. Heparin) } \\
\hline In a stable patient, no organ dysfunction & $10 \%(71)$ & $10 \%(73)$ \\
\hline In a patient with a previous heparin-related hemorrhage & Partial agreement ${ }^{9}$ & $1 \%(71)$ \\
\hline \multicolumn{3}{|l|}{ ELECTROLYTE BOLUS (e.g. Potassium chloride) } \\
\hline In stable patient, no organ dysfunction & $10 \%(66)$ & Partial agreement ${ }^{i}$ \\
\hline
\end{tabular}

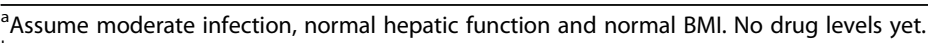

${ }^{\mathrm{b}}$ Assume moderate infection, normal hepatic function and normal BMI. No drug levels yet. Renal dosing adjustment with Creatinine $2 \mathrm{x}$ normal

${ }^{c} N=20$ suggested $>20 \%$ for upper threshold, and $N=12$ suggested $>10 \%, N=4$ blank. $N$ total $=36$.

${ }^{\mathrm{d}} N=19$ suggested $>10 \%$ for upper threshold, and $N=7$ suggested $>10 \%, N=5>50 \%$. $N$ total $=36$.

${ }^{\mathrm{e}} N=6$ would not give drug at all. For overdoing, $N=22(58 \%)$ answered $1-10 \%, N=7(18 \%)$ answered $>10 \%, N=1$ answered $>20 \%, N=1$ blank.

For under-dosing, $N=18(47 \%)$ answered $21-50 \%, N=6(16 \%)$ answered $11-20 \%$.

${ }^{\mathrm{f}} N=6$ would not give drug at all, $N=14$ suggested $>10 \%$ for upper threshold, and $N=9$ suggested $>10 \%$.

${ }^{9} N=3(8 \%)$ answered $1-10 \%, N=21$ answered $>10 \%(58 \%), N=7$ answered $>20 \%(19 \%), N=2$ answered $>50 \%, N=4$ blank or I don't know. $N$ total $=37$.

${ }^{\mathrm{h}}$ Adverse drug event occurred with the drug dose in reference range, and resulted in a 2-day prolongation of hospital stay.

i $N=12$ answered $>10 \%(32 \%), N=21$ answered $>20 \%(57 \%), N=1$ answered $>51 \%, N=3$ blank. $N$ total $=37$.

Consensus not achieved for $2 / 15$ principles.

pediatric dosing resources and definitions, as well as our use of proportions rather than absolute values, will likely make these dosing principles and thresholds clinically valid internationally. Second, we could not exclude neutral response bias of clinicians selecting the same response throughout. Questionnaire burden was a significant concern, given the multiplicity of rounds and survey length. Although we reached over $80 \%$ response rate for each round, study participation took $10-15 \mathrm{~min}$ over the 3 different rounds and required active participation to send back the surveys. We therefore deliberately limited the medication groups and clinical scenarios that were included to limit the respondent burden. Third, the study did not assess continuous infusions of sedatives, analgesics or inotropes commonly used in the PICU, nor did it address rate of administration of intermittent drugs. Fourth, the low nursing participation in the study $(n=4)$ unfortunately limited the weight of nursing respondents in this study. Although most nurses do not prescribe medications in the PICU, they are largely responsible for drug administration. Understanding the subtleties of drug dilution, preparation and administration, their opinions on dose errors would have provided valuable input. Last, although dosing error is made up of prescribing error, dispensing error and administration error; this manuscript does not specifically distinguish between these errors, nor does it address the intentionally of the prescriber. We focused most specifically on administered drug to the patient, i.e. the dose the patient receives, whether the deviation occurred during prescription, 
preparation or administration was not addressed but may have changed response selection. Pharmacy convention however, suggests that a less than $10 \%$ dose deviation during preparation may be acceptable. Prescriber intent further complexifies defining error. If an experienced provider deliberately doses outside refence range and this is not considered an error, then that extended dosing should be reflected in the acceptable principles and thresholds tested in this study (i.e. clinicians can conceive of these acceptable deviations in dosing).

Overall, the consensus driven clinical dosing principles and error thresholds provides useful clinical data to begin to define dosing error in pediatric critical care practice. Further studies are needed to test and refine this $10 \%$ dosing threshold in critical care; including studies of association between 10\% excess dosing and adverse drug events (harm), and prospective studies using adjudication to asses acceptable dosing for different drug classes in various clinical contexts.

\section{Conclusion}

This study provides the first generalizable expert consensus on dosing principles and dosing thresholds in pediatrics. Expert clinical providers in the PICU agreed on the uncertainty in what underlying principles constitute a dosing error, reflecting the complexity in defining it. For most medications and clinical scenarios, dosing over $10 \%$ below or above reference range was considered a dosing error. However, clinical context and drug properties largely influences this threshold. Thresholds may be larger for non-toxic medications, and narrower for very toxic medications, medications with larger side effects or if a previous adverse drug event occurred. These consensus driven findings will help guide prescribing practice, standardized reporting, measurement comparisons in research and drug quality improvement in pediatric critical care.

\section{Supplementary information}

Supplementary information accompanies this paper at https://doi.org/10. 1186/s12887-020-02384-3.

Additional file 1: Table S1. STROBE Checklist. Table S2. Principles of dosing error included in Delphi questionnaire. Figure S1. Example of dosing error threshold question for Round 2.

\section{Abbreviations}

ADE: Adverse Drug Events; CCCTG: Canadian Critical Care Trials Group; ECMO: Extracorporeal Membrane Oxygenation; NSAIDs: Non-Steroidal AntiInflammatory Drugs; PICU: Pediatric Intensive Care Unit

\section{Acknowledgements}

We would like to thank all the healthcare providers in participating PICUs across the country (east to west): Victoria (BC), BC Children's Hospital (BC), Alberta Children's (AB), Stollery Children's (AB), Winnipeg (MN), London Health Sciences (ON), McMaster Children's (ON), Sickkids (ON), Children's
Hospital of Eastern Ontario (ON), Ste-Justine Hospital (QC), Montreal Children's Hospital (QC), Sherbrooke (QC), Quebec (QC), IWK (N-S).

\section{Authors' contributions}

NR conceptualized and designed the study, conducted focus groups, designed the data collection tool, collected all the data, carried out analyses, drafted the manuscript, revised the final manuscript and agrees to be accountable for all aspect of the work. EP helped design the data collection tool (questionnaire), critically revised the manuscript for intellectual content, approved the final version and agrees to be accountable for the work. AT and PR helped conceptualize study, interpret the data, critically reviewed the manuscript for important intellectual content, reviewed the final version and agree to be accountable for all aspects of the work. CP conceptualized and designed the study, conducted focus groups, designed the data collection tool, revised the manuscript and approved the final manuscripts. He agrees to be accountable for all aspects of the work. All authors approved the final manuscript as submitted and agree to be accountable for all aspects of the work.

\section{Funding}

This study was not funded. N. Roumeliotis received doctoral salary support from the Fonds de Recherche Santé- Québec and the Canadian Critical Care Trials Group (CCCTG). Neither of these funding bodies had a role in the study design, collection, analysis and interpretation of the data, or in writing of the manuscript.

\section{Availability of data and materials}

The datasets generated and/or analysed during the current study are not publicly available, but available from the corresponding author [NR] on request.

\section{Ethics approval and consent to participate}

The questionnaire and methodology for this study was approved by the Human Research Ethics committee of the University of Toronto (\#00037969) and the Hospital for Sick Children (\#1000062863). Consent was sought for all participants.

\section{Consent for publication}

The author signs for and accepts responsibility for releasing this material on behalf of any and all co-authors.

\section{Competing interests}

The authors have no conflicts or competing interests relevant to this article to disclose. All authors approved the final manuscript as submitted and agree to be accountable for all aspects of the work.

\section{Author details}

'Department of Critical Care Medicine, The Hospital for Sick Children, 555 University Avenue, Toronto, ON, Canada. ${ }^{2}$ Child Health Evaluative Sciences, SickKids Research Institute, Toronto, Canada. ${ }^{3}$ Dalla Lana School of Public Health, University of Toronto, Toronto, ON, Canada. 'Women's College Research Institute, Women's College Hospital, Toronto, Canada. ${ }^{5}$ Institute of Health Policy, Management and Evaluation, University of Toronto, Toronto, Canada. ${ }^{6}$ Department of Medicine, University of Toronto, Toronto, Canada.

${ }^{7}$ Leslie Dan Faculty of Pharmacy, University of Toronto, Toronto, Canada.

${ }^{8}$ Center for Safety Research, SickKids Research Institute, Toronto, ON, Canada.

Received: 14 August 2020 Accepted: 13 October 2020

Published online: 21 October 2020

References

1. Kaushal R, Bates DW, Landrigan C, McKenna KJ, Clapp MD, Federico F, et al. Medication errors and adverse drug events in pediatric inpatients. Jama. 2001;285(16):2114-20.

2. Kozer E, Scolnik D, Macpherson A, Rauchwerger D, Koren G. The effect of a short tutorial on the incidence of prescribing errors in pediatric emergency care. The Canadian journal of clinical pharmacology $=$. J Can Pharmacol Clin. 2006;13(3):e285-91.

3. Bordun LA, Butt W. Drug errors in intensive care. J Paediatr Child Health. 1992;28(4):309-11. 
4. Fortescue EB, Kaushal R, Landrigan CP, McKenna KJ, Clapp MD, Federico F, et al. Prioritizing strategies for preventing medication errors and adverse drug events in pediatric inpatients. Pediatrics. 2003;111(4 Pt 1):722-9.

5. Wong IC, Ghaleb MA, Franklin BD, Barber N. Incidence and nature of dosing errors in paediatric medications: a systematic review. Drug Saf. 2004;27(9): $661-70$.

6. Howlett MM, Butler E, Lavelle KM, Cleary BJ, Breatnach CV. The impact of technology on prescribing errors in pediatric intensive care: a before and after study. Appl Clin Informatics. 2020;11(2):323-35.

7. Kozer E, Scolnik D, Macpherson A, Keays T, Shi K, Luk T, et al. Variables associated with medication errors in pediatric emergency medicine. Pediatrics. 2002;1 10(4):737-42.

8. Ghaleb MA, Barber N, Dean Franklin B, Wong IC. What constitutes a prescribing error in paediatrics? Qual Safety Health Care. 2005;14(5):352-7.

9. Yu KH, Nation RL, Dooley MJ. Multiplicity of medication safety terms, definitions and functional meanings: when is enough enough? Qual Safety Health Care. 2005;14(5):358-63.

10. Dean B, Barber N, Schachter M. What is a prescribing error? Qual Health Care. 2000;9(4):232-7.

11. Walsh KE, Landrigan CP, Adams WG, Vinci RJ, Chessare JB, Cooper MR, et al. Effect of computer order entry on prevention of serious medication errors in hospitalized children. Pediatrics. 2008;121(3):e421-7.

12. Warrick $C$, Naik $H$, Avis $S$, Fletcher $P$, Franklin $B D$, Inwald D. A clinical information system reduces medication errors in paediatric intensive care. Intensive Care Med. 2011;37(4):691-4.

13. Taylor JA, Loan LA, Kamara J, Blackburn S, Whitney D. Medication administration variances before and after implementation of computerized physician order entry in a neonatal intensive care unit. Pediatrics. 2008; 121(1):123-8.

14. Holdsworth MT, Fichtl RE, Raisch DW, Hewryk A, Behta M, Mendez-Rico E, et al. Impact of computerized prescriber order entry on the incidence of adverse drug events in pediatric inpatients. Pediatrics. 2007;120(5):1058-66.

15. von Elm E, Altman DG, Egger M, Pocock SJ, Gotzsche PC, Vandenbroucke JP. The Strengthening the Reporting of Observational Studies in Epidemiology (STROBE) statement: guidelines for reporting observational studies. Lancet (London, England). 2007;370(9596):1453-7.

16. Fink A, Kosecoff J, Chassin M, Brook RH. Consensus methods: characteristics and guidelines for use. Am J Public Health. 1984;74(9):979-83.

17. McMillan SS, King M, Tully MP. How to use the nominal group and Delphi techniques. Int J Clin Pharm. 2016;38(3):655-62.

18. Howlett MM, Cleary BJ, Breatnach CV. Defining electronic-prescribing and infusion-related medication errors in paediatric intensive care - a Delphi study. BMC Med Inform Decis Mak. 2018;18(1):130.

19. Keeney S. The Delphi technique in nursing and Health Research: WileyBlackwell; 2011.

20. Burns KE, Duffett M, Kho ME, Meade MO, Adhikari NK, Sinuff T, et al. A guide for the design and conduct of self-administered surveys of clinicians. CMAJ. 2008;179(3):245-52.

21. Diamond IR, Grant RC, Feldman BM, Pencharz PB, Ling SC, Moore AM, et al. Defining consensus: a systematic review recommends methodologic criteria for reporting of Delphi studies. J Clin Epidemiol. 2014;67(4):401-9.

\section{Publisher's Note}

Springer Nature remains neutral with regard to jurisdictional claims in published maps and institutional affiliations.

\section{Ready to submit your research? Choose BMC and benefit from:}

- fast, convenient online submission

- thorough peer review by experienced researchers in your field

- rapid publication on acceptance

- support for research data, including large and complex data types

- gold Open Access which fosters wider collaboration and increased citations

- maximum visibility for your research: over $100 \mathrm{M}$ website views per year

At BMC, research is always in progress.

Learn more biomedcentral.com/submissions 